\title{
Prevalence Rate of Hepatitis B Virus in Pregnancy: Implications From a Systematic Review and Meta-Analysis of Studies Published From
}

\section{0 to 2016}

\author{
Masoud Behzadifar, ${ }^{1}$ Nicola Luigi Bragazzi, ${ }^{2}$ Rabeh Movagharnia, ${ }^{3}$ Haniye Sadat Sajadi, ${ }^{4}$ Sanaz
}

Heidarvand Ghelichi, ${ }^{5}$ Morteza Salemi, ${ }^{6}$ Rahim Sohrabi, ${ }^{7}$ Roghayeh Mohammadibakhsh, ${ }^{8}$ Masood Taheri Mirghaed, ${ }^{8}$ Meysam Behzadifar, ${ }^{9}$ Ahmad Ghashghaee, ${ }^{10}$ Wesam Kooti, ${ }^{11}$ Mariano Martini, ${ }^{12}$ Maryam Darvishnia, ${ }^{13}$ Maryam Saran, ${ }^{9}$ and Seyed Moayed Alavian ${ }^{14,}{ }^{*}$

${ }^{1}$ Hepatitis Research Center, Lorestan University of Medical Sciences, Khorramabad, IR Iran

${ }^{2}$ Department of Health Sciences (DISSAL), University of Genoa, Genoa, Italy

${ }^{3}$ Microbiology Department, Azad University of Varamin -Pishva, Tehran, IR Iran

${ }^{4}$ National Institute of Health Research, Tehran University of Medical Sciences, Tehran, IR Iran

${ }^{5}$ Bahrami Pediatric Hospital, Tehran University of Medical Sciences, Tehran, IR Iran

${ }^{6}$ Social Determinants in Health Promotion Research Center, Hormozgan Health Institute, Hormozgan University of Medical Sciences, Bandar Abbas, IR Iran

${ }^{7}$ Iranian Social Security Organization, Zanjan Province Health Administration, Zanjan, IR Iran

${ }^{8}$ Department of Health Services Management, School of Health Management and Information Sciences, Iran University of Medical Sciences, Tehran, IR Iran

${ }^{9}$ Social Determinants of Health Research Center, Lorestan University of Medical Sciences, Khorramabad, IR Iran

${ }^{10}$ Student Research Committee, Department of Health Services Management Branch, School of Health Management and Information Sciences, Iran University of Medical

Sciences, Tehran, IR Iran

${ }^{11}$ Student Research Committee, Kurdistan University of Medical Sciences, Sanandaj, IR Iran

${ }^{12}$ Section of History of Medicine and Ethics, Department of Health Sciences (DISSAL), University of Genoa, Genoa

${ }^{13}$ Department of Nursing, Islamic Azad University Khorramabad Branch, Khorramabad, IR Iran

${ }^{14}$ Research Center for Gastroenterology and Liver Diseases (BRCGL), Baqiyatallah University of Medical Sciences, Tehran, IR Iran

"Corresponding author: Professor. Seyed Moayed Alavian, Baqiyatallah Research Center for Gastroenterology and Liver Diseases (BRCGL), Baqiyatallah University of Medical Sciences, Tehran, IR Iran. Tel: +98-2188945186, Fax: +98-2188945188, E-mail: alavian@thc.ir

Received 2017 June 06; Revised 2017 November 11; Accepted 2017 December 27.

\begin{abstract}
Background: Hepatitis B Virus (HBV) is one of the most serious infectious diseases and represents a major global health issue worldwide. It can be transmitted vertically and horizontally through contact with infected blood or body fluids. More attention to HBV infection in pregnancy is needed due to high risk of chronicity when transmitted to infants during delivery.

Objectives: A comprehensive review of the HBV prevalence rate in pregnant females taking into account different geographical areas and socioeconomic status is still lacking. This would be of crucial importance for HBV prevention and control programs. As such, this systematic review and meta-analysis was conducted focusing on HBV prevalence rate in pregnant females from different parts of the world.

Methods: Different electronic databases, including Embase, PubMed/MEDLINE, Scopus, and ISI/Web of Science were searched from January 1st 2000 to July 31st 2016, using relevant keywords, such as "prevalence" or "seroprevalence" or "epidemiology" and "pregnancy" or "pregnant" or "antenatal" in combination with "hepatitis B virus" or "HBV" with no language restrictions. The study protocol of this systematic review was deposited at the "International Prospective Register of Systematic Reviews" and registered as CRD42016041985.

Results: After scrutinizing all the extant scholarly literature from 2000 to 2016, this study found 222 relevant articles. The overall HBV prevalence rate in pregnant females worldwide was estimated using a random-effect model, giving a value of 3\% (95\% confidence interval or CI $2 \%$ - $4 \%$ ). Heterogeneity between studies was significantly high $\left(\mathrm{I}^{2}=99.9 \%, \mathrm{P}<0.0001\right)$. The clinical and epidemiological burden was higher in developing countries. Conclusions: This suggests that despite the recent scientific advancements and the clinical progress that has occurred in anti-viral therapy, HBV still represents a major issue worldwide, especially in underdeveloped countries. The key strategies for preventing transmission from pregnant females to their fetuses are through early birth dose and infant vaccination, as well as by the use of hepatitis B immunoglobulin (HBIG) and the screening and diagnosis of mothers at high risk and the subsequent use of anti-viral agents during pregnancy in order to reduce maternal DNA concentrations down to undetectable concentrations. Health authorities should effectively implement these approaches to better control HBV in pregnancy.
\end{abstract}

Keywords: Hepatitis B Virus, Pregnancy, Systematic Review and Meta-Analysis, Vaccines and Vaccination

\section{Background}

According to the world health organization (WHO), approximately 240 million people worldwide are affected by chronic hepatitis $(1,2)$. Each year, more than 686,000 pa- tients obtain HBV-related cirrhosis and die of liver cancer (1). In addition, HBV challenges the National health systems and imposes a significant economic burden. Some of the direct costs include the delivery of diagnostic services, 
hospitalization, and the administration of drugs, whilst indirect costs are generated by the loss of quality of life and reduced productivity. The HBV prevalence rate significantly varies among different regions: It is less than $1 \%$ in America and Western Europe, whilst the highest prevalence rate is $5 \%$ to $10 \%$ and can be observed in East Asia and sub-Saharan Africa. A rate between $2 \%$ and $5 \%$ could be found in the Middle East and the Indian subcontinent (2-4).

Hepatitis B Virus can be transmitted both vertically and horizontally through contact with infected blood or body fluids. Risk factors for developing HBV infection include having unprotected sex with multiple sexual partners or with infected individuals, being a man, who has sex with other men, sharing needles during intravenous (IV) drug use, living with infected individuals, being occupationally exposed to blood, and travelling to regions, in which HBV is endemic, among others (5).

Hepatitis B Virus should be seriously taken into consideration during pregnancy, as the infection can have clinical implications for both the mother and the fetus $(6,7)$ : without prophylaxis, the risk of vertical transmission is, indeed, high, ranging from $10 \%$ to $40 \%$ in $\mathrm{HBsAg}$ positive HBeAg-negative mothers to $70 \%$ in $\mathrm{HBs} A g$ - and HBeAg-positive mothers (8). An effective vaccine is currently available, yet, unfortunately, immunization coverage is not very high, especially in developing countries (9). In order to investigate the HBV prevalence rate in pregnant females, many studies have been conducted worldwide. However, to the best of our knowledge, a comprehensive review of the HBV prevalence rate in pregnant female taking into account the different geographical areas and their socio-economic status is still lacking. This would be of crucial importance for HBV prevention and control programs. Recently, in May 2016, the world health organization (WHO) adopted the "Global Health Sector Strategy on Viral Hepatitis, 2016 to 2021" and one of the strategies suggested was "formulating evidence-based policy and data for action" (10).

As such, this systematic review and meta-analysis was conducted in order to systematically collect available data focusing on HBV prevalence rate in pregnant females from different parts of the world. The current findings could have important implications for health policy-and decision-makers as well as for the stakeholders to better understand the current status of HBV in pregnant females.

\section{Methods}

\subsection{Information Sources and Search}

Different electronic databases including Embase, PubMed/MEDLINE, Scopus and ISI/Web of Science were searched from January 1st 2000 to July 31st 2016, using relevant keywords such as "prevalence" or "seroprevalence" or "epidemiology" and "pregnancy" or "pregnant" or "antenatal" in combination with "hepatitis B virus" or "HBV" with no language restrictions. Four authors independently screened titles and abstracts of studies and checked whether they met the inclusion criteria, selecting potentially eligible studies. Any disagreements between reviewers were resolved after discussion involving a fifth referee. This study also consulted the reference list of each potentially relevant study as well as related studies and extant reviews/overviews in order to increase the chance of obtaining all the pertinent studies.

\subsection{Eligibility Criteria}

Inclusion criteria, according to the PICO criteria, were as follows: i) population-based study reporting $\mathrm{HBV}$ prevalence rate in pregnant females with no age limit; ii) studies using a standardized/validated diagnostic test for the detection of $\mathrm{HBV}$ in pregnant females; iii) prevalence rate clearly stated or, if missing, appropriate and relevant quantitative information in order to calculate the prevalence data; iv) full-text available or abstract providing minimum relevant quantitative information; and v) primary research studies published in peer-reviewed journals between January 2000 and July 2016, reporting HBV prevalence rate in pregnant females.

Concerning the exclusion criteria, the research did not select studies with pregnant females from high risk groups, such as drug abusers, Human Immunodeficiency Virus (HIV)-positive pregnant females, females attending sexually-transmitted-disease (STDs) clinics, sex workers and dialysis patients. Further exclusion criteria were: i) studies with unclear prevalence data and/or methodological errors, and ii) non primary research studies.

The methodological quality of the included studies was evaluated using the Newcastle-Ottawa scale (NOS) (11). Two authors independently performed the quality assessment and any disagreements between them were resolved by discussion and/or involving a third person as a judge. Up to four stars were attributed on the basis of selection, up to two stars for study comparability and up to three stars on the basis of the study outcome(s). Studies that received two or three stars and two stars for the selection and comparability items, respectively, were considered as affected by a low or moderate risk of bias, while studies that received a star for the selection, comparability and outcome(s) items or achieved null score in all sections were characterized by a high risk of bias.

From studies meeting the inclusion criteria, the following data were extracted by two independent reviewers: 
namely, surname of the first author, year of study publication, number of women with positive hepatitis B surface antigen (HBsAg), study type, country, sample size, mean age or age range of participants, and HBV prevalence rate. Any disagreements were resolved by discussion. The main outcome of the present study was the HBV prevalence rate in pregnant females. This study was carried out according to the "Preferred Reporting Items for Systematic reviews and Meta-Analysis" (PRISMA) guidelines (12). The study protocol of this systematic review was deposited in the "International Prospective Register of Systematic Reviews" and registered as CRD42016041985 (13).

On the basis of the data provided by the included studies, the pooled prevalence rate with 95\% confidence interval (CI) was calculated using the DerSimonian and Laird random-effect model $(14,15)$. Heterogeneity was estimated carrying out the $\mathrm{I}^{2}$ test (16). In order to evaluate the a priori effects of the pooled HBV prevalence rate in pregnant females, stratified analyses were performed based on factors such as socio-economic status of the study country (countries classified in developed or developing countries according to the International Monetary Fund or IMF), the year of publication of studies, the quality of studies, the geographic areas as classified by the WHO, and mean age or age range of participants (17).

In order to investigate the causes of the heterogeneity between studies, meta-regression analyses were performed based on the sample size and year of publication. To assess the robustness of results, a sensitivity analysis was carried out (18). Studies were ranked according to year of publication and sample size and cumulative metaanalysis was performed to evaluate the impact of these factors (19). Publication bias was determined both visually, inspecting the eventual asymmetry of the funnel plot, and by carrying out the Egger's test (20).

The relationship between pooled HBV prevalence rate in pregnant females and the human development index (HDI) for each studied country was estimated with linear meta-regression. The HDI is a composite index that combines different measures and indicators, such as life expectancy, educational index, and Gross National Income per capita (GNP) as computed by the united national development program (UNDP).

For all the statistical analyses, figures with a Pvalue of $<$ 0.05 were considered as statistically significant. All statistical analyses were carried out using the open-source software R (version 3.3.1, the R Foundation for Statistical Computing, https://www.R-project.org/) with the Meta package.

\section{Results}

3.1. Study Flow-Chart and Characteristics of the Included Studies

Overall, 3149 studies were identified in the initial search, then after removing duplicate items, 2564 studies were assessed and screened regarding the basis of their title and abstract. Finally, 223 studies met the inclusion criteria, with a total sample size of 40,958 , and 838 pregnant females (Figure 1). The main characteristics of included studies are reported in supplementary file Appendix 1, to which the reader is referred for further details.

According to the WHO geographical regions, 79, 46, 30, 28, 23, and 16 studies were carried out in African, European, Eastern Mediterranean, South East Asiatic, American, and Western Pacific areas, respectively.

\subsection{Risk of Bias of Included Studies}

The quality assessment using the NOS instrument showed that 152 and 71 studies were characterized by a low and high risk of bias, respectively. In particular, 174, 195, and 87 studies were characterized by low quality in terms of selection, comparability and outcomes, respectively. Overall, 13, 15 and 21 presented medium quality concerning selection, comparability and outcomes, respectively, whilst 35, 12 and 14 exhibited high quality, for selection, comparability, and outcomes, respectively.

\subsection{Pooled Prevalence Rate of Hepatitis B Virus in Pregnancy}

The overall HBV prevalence rate in pregnant females worldwide, estimated using a random-effect model, was $3 \%$ [95\%CI 2\% - 4\%]. Heterogeneity between studies was significantly high $\left(\mathrm{I}^{2}=99.9 \%, \mathrm{P}<0.0001\right)$ due to epidemiological differences among populations in terms of HBV prevalence, risk factors, and health policies.

\subsection{Results of Sub-Groups Analysis of Included Studies}

Sub-group analyses pooling HBV prevalence rate in pregnant females based on the study country, WHO areas, IMF classification, quality of the published studies, and study design are shown in Table 1.

\subsection{Results of Sensitivity and Cumulative Analyses}

To ensure the robustness of the results, before and after sensitivity analyses were carried out, showing a statistically unchanged prevalence of 3\% [95\%CI 2 to 4\%]. Also, cumulative meta-analyses, based on the ranking of the sample-size and the year of publication of studies, were conducted. The results did not change statistically. 


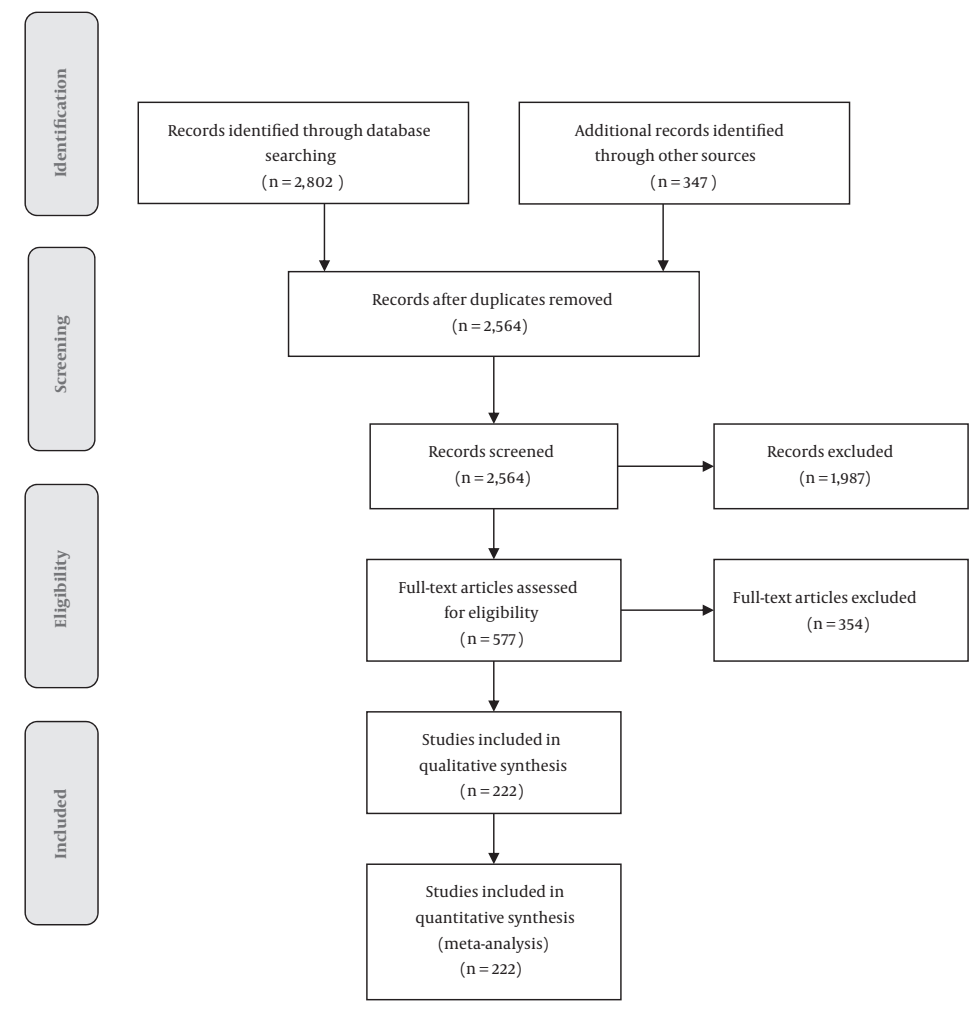

Figure 1. Flow-Chart of the Current Systematic Review and Meta-Analysis, Showing Studies Search and Selection

\subsection{Meta-Regression Analyses}

The findings of the meta-regression analyses based on year of publication and sample-size of the included studies are shown in Table 2 and Figure 2.

\subsection{Publication Bias}

Publication bias analysis showed evidence of publication bias (Egger's test $\mathrm{P}=0.000$ ), as shown in Figure 3 .

\subsection{Relationship between Hepatitis B Virus prevalence and $\mathrm{Hu}$ -} man Development Index

This research found a statistically significant relationship between the pooled HBV prevalence in pregnant females and the HDI of each study country $(\mathrm{R}=-0.12132, \mathrm{P}<$ 0.000) (Figure 4).

\section{Discussion}

Despite recent scientific advancements and clinical progress in anti-viral therapy, HBV still represents a major issue worldwide, especially in developing countries (21, 22). The current study has found a pooled HBV prevalence rate of $3 \%$ [95\%CI $2 \%-4 \%]$ among pregnant females worldwide.

Generally speaking, HBV vaccination represents an effective tool for reducing HBV-related clinical and epidemiological burden. Specifically, focusing on HBV in pregnancy, this infection is particularly unique and challenging. The key strategies for preventing transmission from pregnant females to their fetuses are by early birth dose and infant vaccination, as well as by the use of Hepatitis B Immunoglobulin (HBIG) and the screening and diagnosis of mothers at high risk and the subsequent use of anti-viral agents (such as nucleoside/nucleotide analogues) during pregnancy in order to reduce maternal DNA concentrations to undetectable concentrations (23). Hepatitis B Virus vaccines in pregnancy have been proven safe, with no side-effects in newborns, since no congenital defects or abnormal developmental patterns have been observed. On one hand, HBV immunization during pregnancy is technically and logistically challenging in that the classical schedule (at 0, 1, and 6 months) is difficult to complete given the limited gestational time. On the other hand, accelerated schedules (at 0,1 , and 4 months) have shown an adequate immunological and protective profile. Intra- 
Table 1. Results of Sub-Group Analyses

\begin{tabular}{|c|c|c|c|c|c|}
\hline Variables & Number of Studies & Number of Participants & Poled Prevalence Rate (95\% CI) & $\mathbf{I}^{2}$ & P Value \\
\hline \multicolumn{6}{|l|}{ Continent } \\
\hline Africa & 81 & 79,450 & $6(5-7)$ & 96.4 & $<0.000$ \\
\hline Asia & 78 & 490,372 & $3(2-3)$ & 99.5 & $<0.000$ \\
\hline Europe & 37 & $1,051,387$ & $1(1-2)$ & 99.6 & $<0.000$ \\
\hline South America & 19 & 142,731 & $1(0-2)$ & 99.6 & $<0.000$ \\
\hline North America & 4 & $39,177,247$ & $0(0-1)$ & 99.8 & $<0.000$ \\
\hline Oceania & 3 & 17,651 & $2(2-2)$ & 0 & 0.78 \\
\hline \multicolumn{6}{|c|}{ Country socio-economic status } \\
\hline Developing & 156 & 478,118 & $4(3-4)$ & 98.5 & $<0.000$ \\
\hline Developed & 66 & $40,480,720$ & $1(1-2)$ & 100 & $<0.000$ \\
\hline \multicolumn{6}{|l|}{ Risk of bias } \\
\hline Low risk & 151 & $40,665,166$ & $3(2-4)$ & 99.9 & $<0.000$ \\
\hline High risk & 71 & 293,672 & $3(2-4)$ & 99.2 & $<0.000$ \\
\hline \multicolumn{6}{|l|}{ Year of publication } \\
\hline $2000-2005$ & 24 & 262,676 & $2(1-3)$ & 99.7 & $<0.000$ \\
\hline $2006-2010$ & 54 & 309,111 & $3(2-3)$ & 99.5 & $<0.000$ \\
\hline $2011-2016$ & 144 & $40,387,051$ & $3(2-4)$ & 99.9 & $<0.000$ \\
\hline \multicolumn{6}{|c|}{ WHO regional classification } \\
\hline AFRO & 79 & 76,950 & $6(5-7)$ & 96.3 & $<0.000$ \\
\hline SEARO & 28 & 196,904 & $2(1-3)$ & 99.1 & $<0.000$ \\
\hline EURO & 46 & $1,104,262$ & $1(1-2)$ & 99.5 & $<0.000$ \\
\hline EMRO & 30 & 130,190 & $2(2-3)$ & 95.4 & $<0.000$ \\
\hline PAHO & 23 & $39,319,978$ & $1(0-1)$ & 99.9 & $<0.000$ \\
\hline WPRO & 16 & 130,554 & $6(5-8)$ & 99.2 & $<0.000$ \\
\hline \multicolumn{6}{|l|}{ Type of study } \\
\hline Case - control & 5 & 1,995 & $5(3-9)$ & 80.8 & 0.0003 \\
\hline Cohort & 2 & 6,295 & $7(7-8)$ & 0 & 0.3642 \\
\hline Cross-sectional & 188 & $1,508,658$ & $3(3-4)$ & 99.5 & 0.0001 \\
\hline Prospective & 7 & 18,795 & $3(2-6)$ & 96.3 & 0.0001 \\
\hline Retrospective & 20 & $39,423,095$ & $1(0-3)$ & 100 & 0.0001 \\
\hline
\end{tabular}

Table 2. Results of Meta-Regression Analyses

\begin{tabular}{lccccc}
\hline Variable & Estimated & Standard Error & Z Value & P Value & Lower CI95\% \\
\hline Year & 0.0277 & 0.0332 & 0.8341 & 0.4042 & -0.0374 \\
Sample-size & -0.000 & 0.000 & -2.3775 & 0.0174 & -0.0000 \\
\hline
\end{tabular}

dermal route is another promising approach that could be used in high-risk patients, such as subjects with celiac disease (24) as well as in non-responder individuals in order to accelerate the formation of an immunological response and exhibit a protective and adequate safety profile even in pregnancy (25). However, different surveys have underlined low maternal vaccine uptake and have found that many misconceptions and prejudices about HBV in preg- 

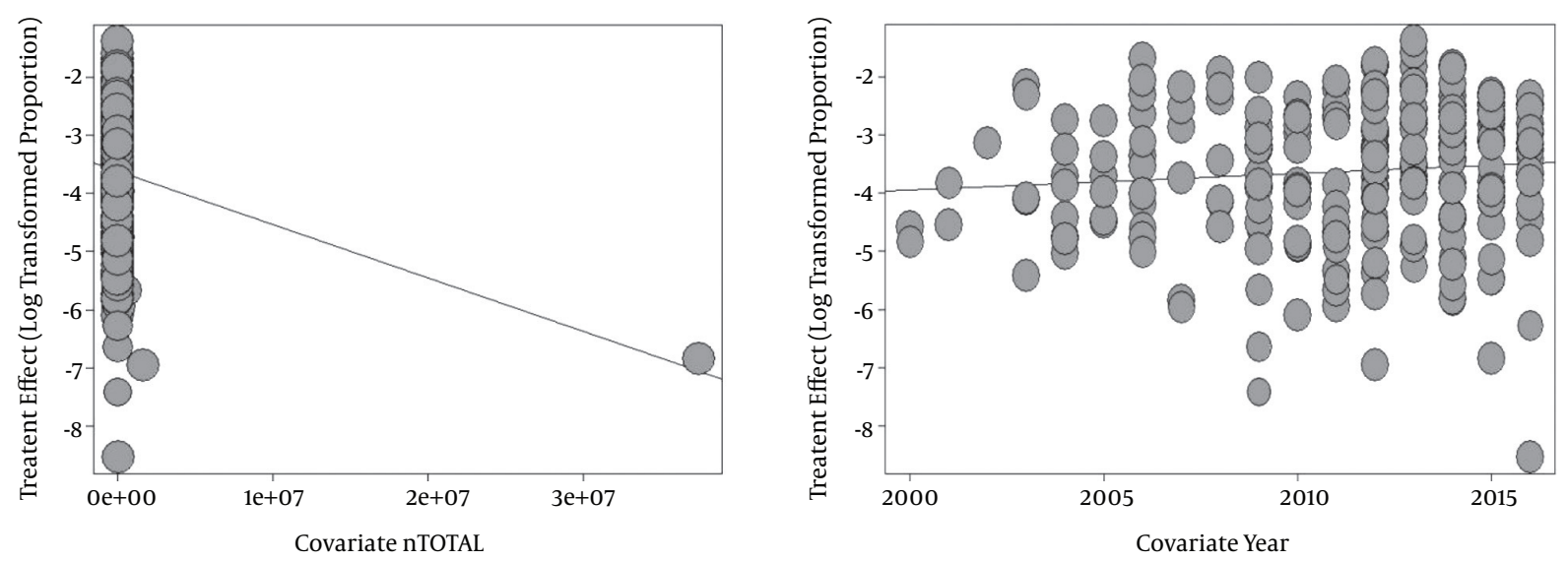

Figure 2. Results of the Meta-Regression Analyses Based on the Sample-Size and Year of Publication of the Included Studies
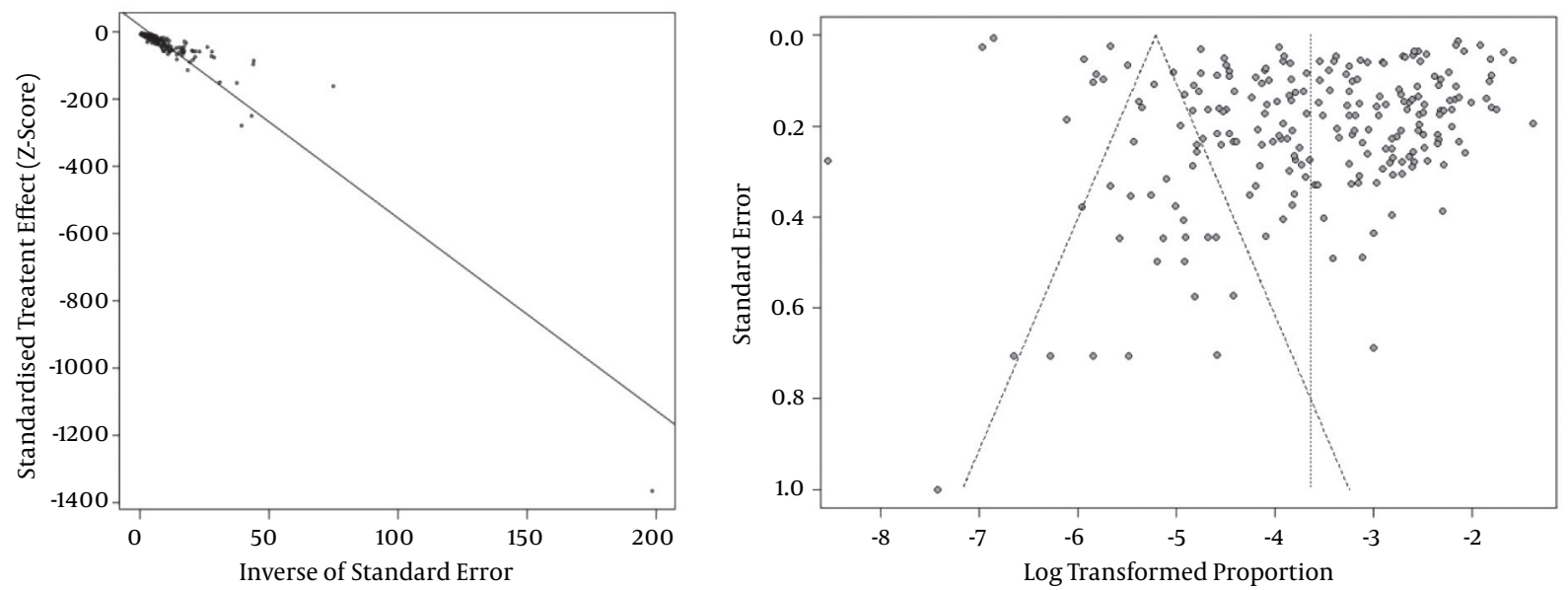

Figure 3. Publication Bias Analyses (Funnel Plot and Egger's Test)

nancy do exist. Educational interventions have focused on benefits to both maternal and neonatal health, which should be made in order to increase maternal knowledge related to $\mathrm{HBV}$ for improving vaccine confidence and, subsequently, vaccine coverage (7).

Furthermore, infant vaccination is particularly recommended in countries characterized by high HBV endemicity. Concerning anti-virals, according to a recently published meta-analysis, pharmacological treatment is able to effectively reduce peri-natal or mother-to-child transmission, as defined by infant HBSAg seropositivity (risk ratio or $\mathrm{RR}=0.3$ [95\%CI $0.2-0.4]$ ) or infant HBV DNA seropositivity $(\mathrm{RR}=0.3[95 \% \mathrm{CI} 0.2-0.5])$ at 6 to 12 months, with no side-effects (in terms of congenital malformation rate, pre- maturity rate, and Apgar score) (26).

Combined, multifaceted strategies integrating screening, prevention, and treatment have been proven to be effective in reducing and mitigating HBV-related burden (27-30). As such, health authorities should effectively implement programs and approaches to increase maternal health literacy and to better control HBV in pregnancy. Furthermore, researchers should design high-quality investigations, covering this topic, which is often underestimated and overlooked in the extant literature.

Decision- and policy-makers should take into account the context and the setting for better design and implementation of ad hoc interventions and strategies. The findings of this study showed, indeed, a significant relation- 


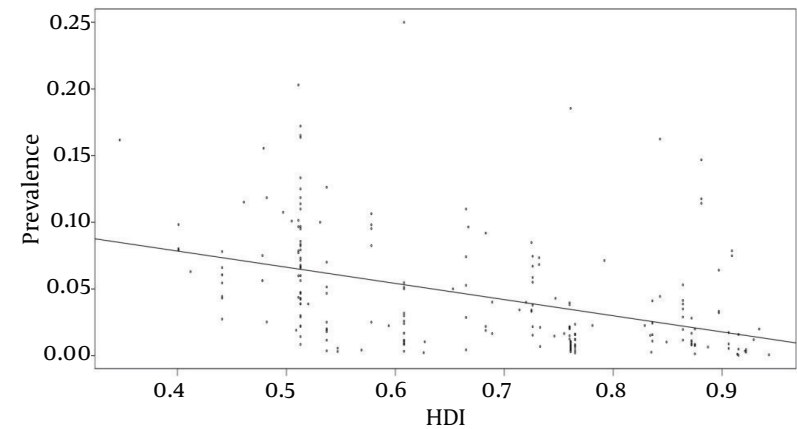

Figure 4. Correlation Between the Pooled Hepatitis B Virus Prevalence in Pregnant Females and the Human Development Index for Each Study Country

ship between HDI and prevalence of HBV among pregnant females ( $\mathrm{P}<0.05$ ). Furthermore, HDI, as introduced by the UND, is a standardized summary measure of social and economic development of countries (31). In many studies, HDI has been found to be a robust, reliable predictor of maternal and infant mortality rate, worldwide (32). In different studies, included in the present systematic review and meta-analysis, maternal high educational levels correlated with a higher awareness of baby's health and resulted in healthier behaviors during pregnancy and delivery, such as attending primary care services and performing HBV screening (33). Low socio-economic status was significantly associated with higher HBV prevalence rate and lower screening adhesion among pregnant females (34, 35).

Concerning the current study, this systematic review and meta-analysis, despite having different strengths like the pre-designed protocol, comprehensive and broad database search, use of different independent reviewers and data extractors, and a rigorous methodological approach, had some drawbacks and limitations that should be acknowledged. These include the high heterogeneity between studies and the fact that approximately a third of the included studies presented high risk of bias.

\subsection{Conclusions}

Our systematic review and meta-analysis has scrutinized all the scholarly literature from 2000 to 2016 and has captured all the relevant studies reporting HBV prevalence in pregnant females. This study has practical implications for policy- and decision-makers, warranting the adoption and implementation of ad hoc programs and strategies based on epidemiological data and on scientific evidences.

\section{Supplementary Material}

Supplementary material(s) is available here [To read supplementary materials, please refer to the journal website and open PDF/HTML].

\section{References}

1. World Health Organization (WHO). Hepatitis B,Fact sheet 2016. Geneva, Switzerland: World Health Organization; 2016. Available from: http: //www.who.int/mediacentre/factsheets/fs204/en/.

2. Salehi Vaziri M, Sadeghi F, Almasi Hashiani A, Gholami Fesharaki M, Alavian SM. Hepatitis B virus infection in the general population of iran, an updated systematic review and meta analysis. Hepat Mon. 2016;16(4):35577. doi: 10.5812/hepatmon.35577. [PubMed: 27257428].

3. Babanejad M, Izadi N, Najafi F, Alavian SM. The HBsAg prevalence among blood donors from eastern mediterranean and middle eastern countries, a systematic review and meta analysis. Hepat Mon. 2016;16(3):35664. doi: 10.5812/hepatmon.35664. [PubMed: 27226804].

4. Zampino R, Sagnelli C, Boemio A, Sagnelli E, Coppola N. Treatment of chronic HBV infection in developing countries. Ann Hepatol. 2016;15(6):816-23. doi: 10.5604/16652681.1222096. [PubMed: 27740514].

5. Chan SL, Wong VW, Qin S, Chan HL. Infection and Cancer: The Case of Hepatitis B. J Clin Oncol. 2016;34(1):83-90. doi: 10.1200/JCO.2015.61.5724. [PubMed: 26578611].

6. Rukunuzzaman M, Afroza A. Risk factors of hepatitis B virus infection in children. Mymensingh Med J. 2011;20(4):700-8. [PubMed: 22081192].

7. Kabir A, Alavian SM, Ahanchi N, Malekzadeh R. Combined passive and active immunoprophylaxis for preventing perinatal transmission of the hepatitis B virus in infants born to HBsAg positive mothers in comparison with vaccine alone. Hepatol Res. 2006;36(4):265-71. doi: 10.1016/j.hepres.2006.08.001. [PubMed: 16965939].

8. Gentile I, Borgia G. Vertical transmission of hepatitis B virus: challenges and solutions. Int J Womens Health. 2014;6:605-11. doi: 10.2147/IJWH.S51138. [PubMed: 24966696].

9. Nelson NP, Easterbrook PJ, McMahon BJ. Epidemiology of hepatitis B virus infection and impact of vaccination on disease. Clin Liver Dis. 2016;20(4):607-28. doi: 10.1016/j.cld.2016.06.006. [PubMed: 27742003].

10. World Health Organization (WHO). Global health sector strategy on viral hepatitis 2016-2021. Geneva, Switzerland: World Health Organization; 2016. Available from: http://www.who.int/hepatitis/ strategy2016-2021/ghss-hep/en/.

11. Stang A. Critical evaluation of the Newcastle-Ottawa scale for the assessment of the quality of nonrandomized studies in meta-analyses. Eur J Epidemiol. 2010;25(9):603-5. doi: 10.1007/s10654-010-9491-z. [PubMed: 20652370].

12. Liberati A, Altman DG, Tetzlaff J, Mulrow C, Gotzsche PC, Ioannidis JP, et al. The PRISMA statement for reporting systematic reviews and meta analyses of studies that evaluate healthcare interventions, explanation and elaboration. BMJ. 2009;339:2700. doi: 10.1136/bmj.b2700. [PubMed: 19622552].

13. Behzadifar M BMSHTMMFZMBR. Prevalence of hepatitis $B$ in pregnancy: a systematic review and meta-analysis. University of York, UK: PROSPERO International prospective register of systematic reviews, CRD42016041985; 2016. Available from: www.crd.york.ac.uk/ PROSPERO/display_record.asp?ID=CRD42016041985..

14. DerSimonian R, Laird N. Meta-analysis in clinical trials. Control Clin Trials. 1986;7(3):177-88. [PubMed: 3802833].

15. DerSimonian R. Combining evidence from clinical trials. Anesth Analg. 1990;70(5):475-6. [PubMed: 2139548]. 
16. Higgins JP, Thompson SG, Deeks JJ, Altman DG. Measuring inconsistency in meta-analyses. BMJ. 2003;327(7414):557-60. doi: 10.1136/bmj.327.7414.557. [PubMed: 12958120].

17. Baker WL, White CM, Cappelleri JC, Kluger J, Coleman CI, Health Outcomes $\mathrm{P}$, et al. Understanding heterogeneity in meta analysis: the role of meta regression. Int J Clin Pract. 2009;63(10):1426-34. doi: 10.1111/j.1742-1241.2009.02168.x. [PubMed: 19769699].

18. Thabane L, Mbuagbaw L, Zhang S, Samaan Z, Marcucci M, Ye C, et al. A tutorial on sensitivity analyses in clinical trials: the what, why, when and how. BMCMed Res Methodol.2013;13:92. doi:10.1186/1471-2288-13-92. [PubMed: 23855337].

19. Leimu R, Koricheva J. Cumulative meta-analysis: a new tool for detection of temporal trends and publication bias in ecology. Proc Biol Sci. 2004;271(1551):1961-6. doi: 10.1098/rspb.2004.2828. [PubMed: 15347521].

20. Egger M, Davey Smith G, Schneider M, Minder C. Bias in metaanalysis detected by a simple, graphical test. BMJ.1997;315(7109):62934. [PubMed: 9310563].

21. Jonas MM. Hepatitis B and pregnancy: an underestimated issue. Liver Int. 2009;29 Suppl 1:133-9. doi: 10.1111/j.1478-3231.2008.01933.x. [PubMed: 19207977].

22. Pawlowska M, Pniewska A, Pilarczyk M, Kozielewicz D, Domagalski K. Prophylaxis of vertical HBV infection. Expert Opin Drug Saf. 2016;15(10):1361-8. doi: 10.1080/14740338.2016.1211106. [PubMed: 27402246].

23. Visvanathan K, Dusheiko G, Giles M, Wong ML, Phung N, Walker S, et al. Managing HBV in pregnancy. Prevention, prophylaxis, treatment and follow-up: position paper produced by Australian, UK and New Zealand key opinion leaders. Gut. 2016;65(2):340-50. doi: 10.1136/gutjnl-2015-310317. [PubMed: 26475631].

24. Filippelli M, Lionetti E, Pulvirenti A, Gennaro A, Lanzafame A, Marseglia GL, et al. New approaches in hepatitis B vaccination for celiac disease. Immunotherapy. 2014;6(8):945-52. doi: 10.2217/imt.14.64. [PubMed: 25313572].

25. Filippelli M, Lionetti E, Gennaro A, Lanzafame A, Arrigo T, Salpietro $C$, et al. Hepatitis B vaccine by intradermal route in non responder patients: an update. World J Gastroenterol. 2014;20(30):10383-94. doi: 10.3748/wjg.v20.i30.10383. [PubMed: 25132754].

26. Bonanni P. Universal hepatitis B immunization, infant, and in- fant plus adolescent immunization. Vaccine. 1998;16 Suppl:17-22. [PubMed: 9915027].

27. Kalantari H, Davari M, Akbari M, Hejazi SM, Kalantari M, Zakerin $\mathrm{S}$, et al. The estimation of direct medical costs of treating patients with chronic hepatitis B and C in iran. Int J Prev Med. 2012;3(3):191-6. [PubMed: 22448312].

28. Lu J, Xu A, Wang J, Zhang L, Song L, Li R, et al. Direct economic burden of hepatitis B virus related diseases: evidence from Shandong, China BMC Health Serv Res. 2013;13:37. doi: 10.1186/1472-6963-13-37. [PubMed 23368750].

29. G. B. D. Mortality, Causes of Death C. Global, regional, and national age sex specific all cause and cause specific mortality for 240 causes of death, 1990-2013, a systematic analysis for the global burden of disease study 2013. Lancet. 2015;385(9963):117-71. doi: 10.1016/S01406736(14)61682-2. [PubMed: 25530442].

30. Brown RJ, McMahon BJ, Lok AS, Wong JB, Ahmed AT, Mouchli MA et al. Antiviral therapy in chronic hepatitis B viral infection during pregnancy: A systematic review and meta-analysis. Hepatology 2016;63(1):319-33. doi:10.1002/hep.28302. [PubMed: 26565396].

31. United Nations Development Programme (UNDP). Human development report 2010, the real wealth of nations, pathways to human development. New York, United States: United Nations Development Programme, (UNDP); 2013, [cited 23 Sep]. Available from: http://hdr.undp. org/en/reports/global/hdr2010/.

32. Lee KS, Park SC, Khoshnood B, Hsieh HL, Mittendorf R. Human development index as a predictor of infant and maternal mortality rates. Pediatr. 1997;131(3):430-3. [PubMed: 9329421].

33. Wang AL, Qiao YP, Wang LH, Fang LW, Wang F, Jin X, et al. Integrated prevention of mother-to-child transmission for human immunodeficiency virus, syphilis and hepatitis B virus in China. Bull World Health Organ. 2015;93(1):52-6. doi: 10.2471/BLT.14.139626. [PubMed: 25558108].

34. Chen L, Evans T, Anand S, Boufford JI, Brown H, Chowdhury $M$, et al. Human resources for health: overcoming the crisis. Lancet. 2004;364(9449):1984-90. doi: 10.1016/S0140-6736(04)17482-5. [PubMed: 15567015].

35. Anand S, Barnighausen T. Human resources and health outcomes: cross-country econometric study. Lancet. 2004;364(9445):1603-9. doi 10.1016/S0140-6736(04)17313-3. [PubMed: 15519630]. 